\title{
Characterisation of a new Fast CPC and its application for atmospheric particle measurements
}

\author{
B. Wehner, H. Siebert, M. Hermann, F. Ditas, and A. Wiedensohler \\ Leibniz Institute for Tropospheric Research, 04318 Leipzig, Germany \\ Received: 13 December 2010 - Published in Atmos. Meas. Tech. Discuss.: 21 December 2010 \\ Revised: 30 March 2011 - Accepted: 11 April 2011 - Published: 6 May 2011
}

\begin{abstract}
A new Fast CPC (FCPC) using butanol as working fluid has been built based on the setup described by Wang et al. (2002). In this study, we describe the new instrument. The functionality and stable operation of the FCPC in the laboratory, as well as under atmospheric conditions, is demonstrated. The counting efficiency was measured for three temperature differences between FCPC saturator and condenser, 25,27 , and $29 \mathrm{~K}$, subsequently resulting in a lower detection limit between 6.1 and $8.5 \mathrm{~nm}$. Above $25 \mathrm{~nm}$ the FCPC reached $98-100 \%$ counting efficiency compared to an electrometer used as the reference instrument. The FCPC demonstrated its ability to perform continuous measurements over a few hours in the laboratory with respect to the total particle counting. The instrument has been implemented into the airborne measurement platform ACTOS to perform measurements in the atmospheric boundary layer. Therefore, a stable operation over two hours is required. The mixing time of the new FCPC was estimated in two ways using a time series with highly fluctuating particle number concentrations. The analysis of a sharp ramp due to a concentration change results in a mixing time of $5 \mathrm{~ms}$ while a spectral analysis of atmospheric data demonstrates that for frequencies up to $10 \mathrm{~Hz}$ coherent structures can be resolved before sampling noise dominates.
\end{abstract}

\section{Introduction}

Condensation Particle Counters (CPCs) are the most widely used instruments for measuring submicrometer aerosol particle number concentrations. Inside the CPC, particles which are too small to be detected by direct optical means, grow due to the condensation of a working fluid. Afterwards, they are

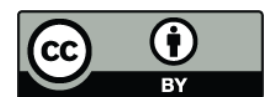

Correspondence to: $\mathrm{B}$. Wehner (birgit@tropos.de) detected by a laser-photodiode optics. Generally, there are three types of CPCs: (1) adiabatic expansion, (2) conductive cooling or continuous flow, and (3) mixing type (Baron and Willeke, 2001). In atmospheric research, the continuous flow type CPC is most frequently used, because of its robustness and reliability. The typical temporal resolution of the simpler unsheathed CPCs is on the order of seconds (Quant et al., 1992; Buzorius, 2001; Held and Klemm, 2006). This "low" temporal resolution is caused by zones of recirculation and the relatively large time required to establish the supersaturation in the laminar flow condenser (Wang et al., 2002).

In atmospheric science, many open questions about particle dynamics require highly time-resolved particle measurements. The spectrum covers vertical turbulent particle fluxes (e.g., the covariance of the vertical velocity and aerosol number concentration), thin stratified layers of increased particle number concentrations, as well as new particle formation due to nucleation and growth connected with turbulent mixing. Enhanced concentrations of small particles have been observed around clouds (Hoppel et al., 1994; Weber et al., 2001) or around the inversion layer due to the mixing processes (Stratmann et al., 2003; Siebert et al., 2004). All these phenomena can occur on small spatial scales or are based on atmospheric fluctuations due to the turbulence of the underlying velocity field (Wehner et al., 2010).

Although ground-based measurements of atmospheric particle fluxes were reported in the past (Buzorius et al., 1998, 2001; Held and Klemm, 2006; Klemm et al., 2006), measurements with higher temporal resolution are required to resolve the high frequency part of the covariance. In previous experiments, this high frequency part has been ignored leading to an underestimation of the vertical aerosol flux. This is also true, in particular, for airborne measurements where the requirements for the sampling frequency are even higher to resolve small structures. Nucleation around clouds was mainly observed by fast-flying aircraft (Weber et al., 2001); with a time resolution in the order of one second

Published by Copernicus Publications on behalf of the European Geosciences Union. 
the spatial resolution resulting from the true air speed of the aircraft $\left(\sim 100 \mathrm{~m} \mathrm{~s}^{-1}\right)$ is $100 \mathrm{~m}$. New particle formation may be a locally restricted phenomenon and, thus, aircraft-borne systems received only a few measurement points in the region where new particles were formed.

Airborne particle measurements with a higher spatial resolution can be achieved by two ways: (1) reduction of the true airspeed (TAS) of the aircraft, and (2) development of faster instrumentation. The first option has already been realised by choosing a helicopter operating with a TAS of $20 \mathrm{~m} \mathrm{~s}^{-1}$, which improves the spatial resolution by a factor of five compared with a typical research aircraft with a typical TAS of $\sim 100 \mathrm{~m} \mathrm{~s}^{-1}$. To increase the temporal resolution of aerosol measurements, the development of so-called "mixing-type CPCs" was started in the eighties (Okuyama et al., 1984). In these instruments, a cold aerosol flow is mixed with a warmer saturated gas flow. In a demonstration study, Wang et al. (2002) showed that mixing times as small as $0.06 \mathrm{~s}$ can be achieved with a new design, which is at least 10 times faster than any commercial unsheathed CPC.

Besides the advantage of having fast CPCs as stand-alone instruments, they are also very useful as part of a Scanning Mobility Particle Sizers (SMPS) system, which measures the particle size distribution. The currently used SMPS systems are limited in time resolution to about $2 \mathrm{~min}$, mainly caused by the slow response of the CPC. With their fast mixing CPC, Wang et al. (2002) have shown that scan times down to $3 \mathrm{~s}$ might be possible under certain conditions. However, in the atmosphere the limiting factor is mostly the number concentration due to counting statistics, thus, atmospheric background measurements will probably not allow scan times shorter than $30 \mathrm{~s}$.

For fast particle measurements, the usage of mixing type CPCs has, therefore, been considered and tested in different studies. However, a continuous and stable operation under atmospheric conditions has not been reported yet for a fast mixing type CPC. Recently, Vanhanen et al. (2011) used the principle of particle growth due to turbulent mixing with a saturated gas flow to measure aerosol particles down to $1 \mathrm{~nm}$. During a field study over several days, its functionality for ambient measurements was demonstrated. In this investigation, we developed and characterised a new Fast CPC (FCPC), which is designed for airborne applications in the atmospheric boundary layer. In the following section, we describe the assembly of the FCPC. Thereafter, the dependency of the counting efficiency on the temperature difference between saturator and condenser, its counting stability as well as the time resolution will be presented and discussed.

\section{Setup}

The Fast CPC (FCPC) presented here is based on the principle of the mixing-type CPC described in Wang et al. (2002) and is shown in Fig. 1. Basically, a warm butanol-saturated

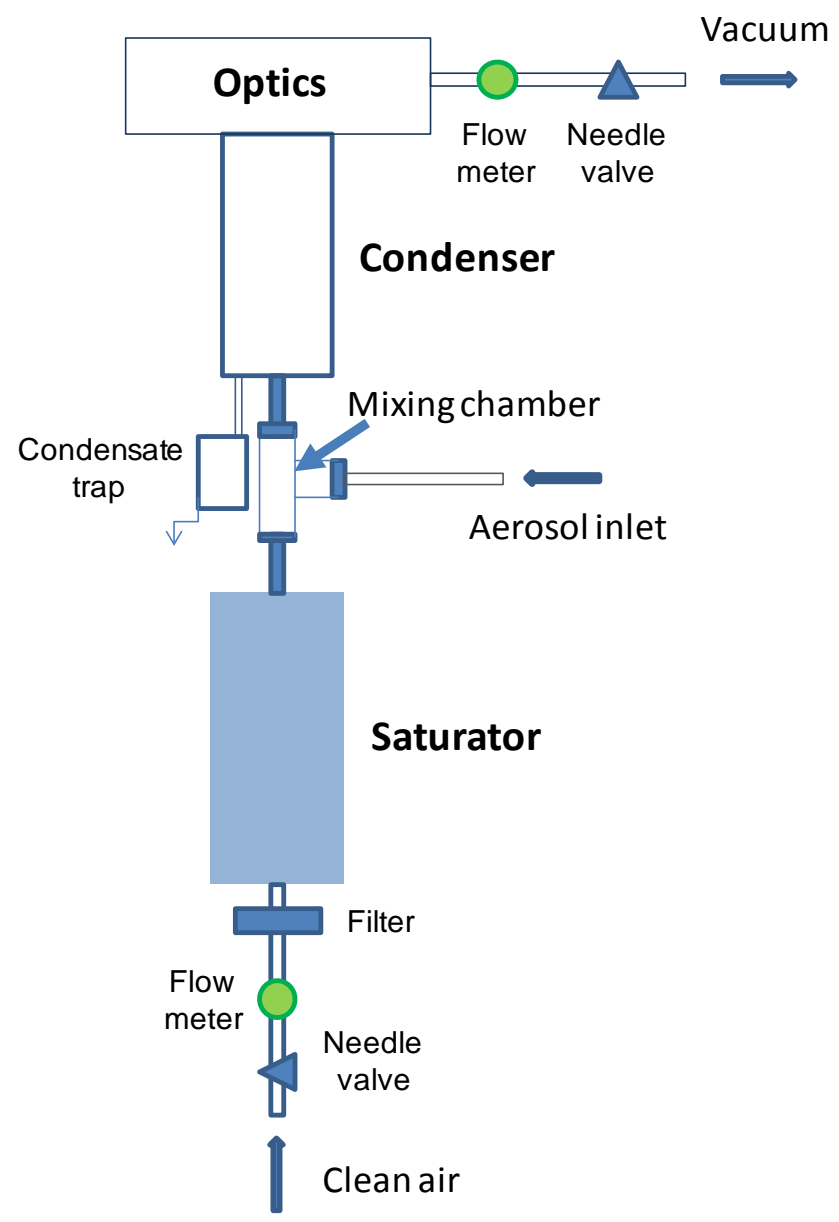

Fig. 1. Schematic of the new Fast CPC. A warm butanol-saturated air flow is mixed with the aerosol flow inside a Swagelok "T". In the following condenser block the air is cooled, causing the required growth of aerosol particles to be counted inside the optics.

air flow is mixed with the aerosol flow within a small mixing volume. Before entering the saturator, the air flow is pressed through a filter and controlled by a needle valve. The saturator contains a wick, which is soaked with liquid butanol during operation. Furthermore, the saturator is equipped with a heating foil and a temperature sensor to provide a defined temperature inside. Similar to Wang et al. (2002), who used a $1 / 4$ inch Swagelok cross in our case a 1/4 inch Swagelok "T" is used as the mixing chamber. We tried both options: the Swagelok cross and "T" with similar results. The "T" version was finally chosen because it saves space in comparison to the cross version. In the following condenser block, the air is cooled causing the required growth of aerosol particles. The Swagelok "T" is isolated and decoupled thermally from the condenser block by a teflon cartridge to avoid cooling of the saturator flow before mixing.

The condenser block downstream of the mixing chamber is cooled by a Peltier element and again temperaturecontrolled by a temperature sensor. The inner diameter of the condenser was decreased to $3 \mathrm{~mm}$ to minimize the residence 
time there compared to other CPCs. Because butanol is continuously condensing on the walls of the condenser, a vacuum flow of $0.21 \mathrm{~min}^{-1}$ is connected through a condensation trap to remove any condensed butanol. The butanol droplets are counted in a standard optics from a TSI CPC 3772 . The temperatures within the saturator $\left(T_{\mathrm{S}}\right)$ and the condenser $\left(T_{\mathrm{C}}\right)$ can be varied and were changed during the calibration procedure. The reason to do that is to shift the lower measurement limit, i.e., the $50 \%$ detection efficiency diameter $\left(D_{\mathrm{P} 50}\right)$. The cooled condenser block is different from the setup described in Wang et al. (2002) and other mixing-type CPCs described in the literature, where no active cooling is applied. Therefore, we do not call our instrument "mixing-type CPC", but the operation principle is somehow similar. The advantage of this chosen setup is the lower temperature difference saving power for cooling and heating which is an important point for airborne applications. Another advantage for our purpose is the stable temperature difference between saturator and condenser which is in fact not possible for real mixingtype CPCs without controlling the inlet temperature because usually ambient temperatures may vary.

Aerosol and saturator flow rate of $0.31 \mathrm{~min}^{-1}$ and $0.71 \mathrm{~min}^{-1}$ were found to fulfil the requirements concerning counting statistics, short residence times and stable operation best. The flows through the saturator and optics were adjusted by needle valves and are monitored continuously during operation. The FCPC was designed to be applied at airborne applications, therefore, no liquid butanol, which is not soaked by the wick, should be within the instrument. The typical duration of helicopter-borne measurement flights is usually about two hours, therefore, filling the wick is sufficient and no continuous butanol filling is required. The absence of liquid butanol in the instrument reduces the number of potential wrong counting during flight. The presented field version is the result of a few-year process. The development included also half a dozen laboratory versions which did not fulfil the requirements sufficiently. Thus, for instance a horizontally-oriented saturator and a condenser without butanol-removal did not allow for a stable operation under atmospheric conditions.

\section{Characterisation of the Fast CPC}

\subsection{Counting efficiency}

The particle counting efficiency curve was determined according to the setup described by Hermann et al. (2007); Hermann and Wiedensohler (2001); Wiedensohler et al. (1997). Here, polydisperse silver aerosol was generated in a tube furnace at temperatures of $1050-1150^{\circ} \mathrm{C}$ according to Scheibel and Porstendörfer (1983). Downstream of the furnace, a dilution system was used to keep the particle number concentration at the CPCs between 1000 and 5000 particles $\mathrm{cm}^{-3}$. This range was chosen in order to have a good signal-to-noise ratio at the reference electrometer (EM, TSI 3068B) and to minimize coincidental effects in the CPC optics. After dilution, the aerosol particles were charged in an ${ }^{85} \mathrm{Kr}$ bipolar charger (Wiedensohler, 1988) and a monodisperse fraction was selected by a differential mobility analyser (DMA, Vienna-type, short: $L=11 \mathrm{~cm}, r_{\text {in }}=2.5 \mathrm{~cm}, r_{\text {out }}=3.35 \mathrm{~cm}$ ). The sheath and excess air flow rates of the DMA were adjusted to $201 \mathrm{~min}^{-1}$, the aerosol and sampling flow rate was set to $21 \mathrm{~min}^{-1}$. Downstream of the DMA, the monodisperse aerosol was diluted with particle-free air to achieve the required total flow rate for the particle counter(s) and the aerosol electrometer as reference instrument. The individual instruments were fed from a $2 \mathrm{~m}$ long tube header with 11 ports downstream of the DMA. Sampling lines from the header to the instruments were arranged in a symmetrical pattern each with a $50 \mathrm{~cm}$ straight line. The EM flow rate was $21 \mathrm{~min}^{-1}$ and the EM data were corrected for background offset, measured regularly between the particle measurements. The different sampling flows of FCPC and EM cause differences in diffusional losses mainly below $20 \mathrm{~nm}$. The presented values are corrected for these losses. During our study, the temperature in the condenser of the FCPC was kept constant at $10^{\circ} \mathrm{C}$ while the saturator temperature was set to $35^{\circ} \mathrm{C}, 37^{\circ} \mathrm{C}$, and $39^{\circ} \mathrm{C}$. These settings were chosen because of stable and reliable FCPC performance in the laboratory and in the atmosphere. For much higher temperature differences, the measurements became unstable and for much lower temperature differences the counting efficiency went down.

The counting efficiency was measured for diameters between 5 and $30 \mathrm{~nm}$, the counting time at each selected diameter was $5 \mathrm{~min}$. The calibration curves were measured at least three times for each temperature difference and mean values were calculated. The FCPC particle counting efficiency $\eta$ was finally calculated as the ratio of the mean FCPC and EM particle number concentrations at each individual particle diameter $D_{\mathrm{P}}$.

Figure 2 shows the mean values for the FCPC at the three different temperature settings. Uncertainties in the particle diameter selected by the DMA are mainly caused by deviations from the chosen flow ratio and are less than $2 \%$ for this setup. A systematic error in particle size is caused due to the shape of the size distribution produced by the tube furnace. For our measurements, particle sizes larger than the maximum of the size distribution were chosen in order to minimize the effect of multiple charges on the EM reading. Due to the skewing and the width of the DMA transfer function, the maximum of the selected particles size is slightly shifted to smaller sizes not more than $2 \%$ in our case. Uncertainties in the counting efficiency result from the standard deviations of the mean counting efficiencies at each diameter. For the FCPC the standard deviation was usually below $5 \%$ of the counting efficiency, but in the cutoff region it can be higher (up to $10 \%$ ). 


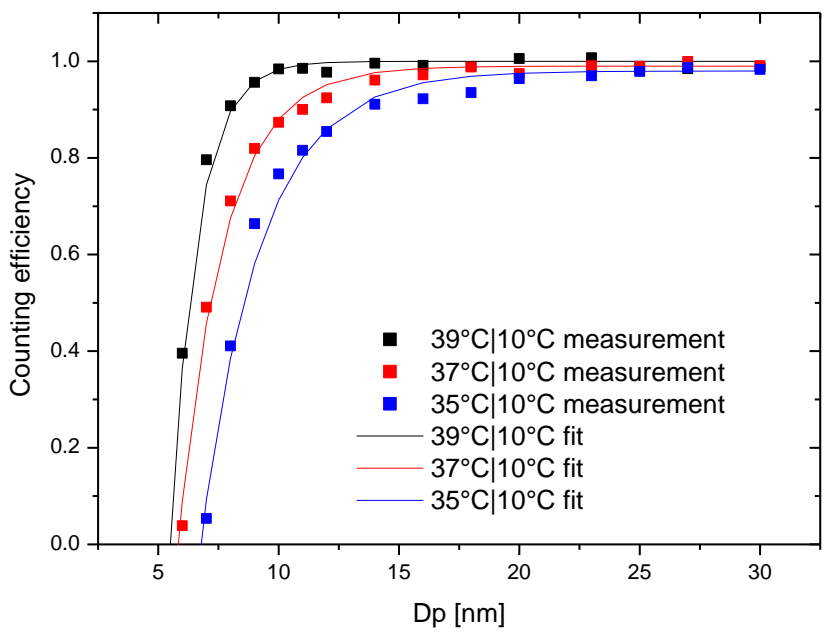

Fig. 2. Counting efficiency of the Fast CPC at different temperature differences compared to the electrometer (TSI 3068B). Squares present the mean measurement points, the solid lines are fitted using Eq. (1). The corresponding fit parameters as well as the 50\% detection diameter $D_{\mathrm{P} 50}$ are given in Table 1 .

The counting efficiency data were fitted using a threeparameter exponential function according to Wiedensohler et al. (1997):

$\eta=a-\exp \left(\frac{D_{1}-D_{\mathrm{P}}}{D_{2}}\right)$.

With the help of this empirical approach, the diameters of the $50 \%$ particle detection efficiency, $D_{\mathrm{P} 50}$, were calculated. In this context, the $D_{\mathrm{P} 50}$ was defined as particle diameter where the counting efficiency reaches half the maximum of the reference instrument reading $(100 \%)$. The fitting parameters as well as the $D_{\mathrm{P} 50}$ values are summarized in Table 1 . The parameter $a$ describes the maximum value which is reached by the counting efficiency curve. Usually it should be close to 1 which is also the case for the FCPC. The two parameters $D_{1}$ and $D_{2}$ are characteristic diameters for each curve: $D_{1}$ is close to $D_{\mathrm{P} 50}, D_{2}$ is significantly lower and may be considered as the diameter where the FCPC does not count any particles. Both diameters are shifted to lower sizes with increasing temperature difference, as expected (Banse et al., 2001). The three curves are similar in shape. For the given temperature settings, the $D_{\mathrm{P} 50}$ varies between 6.1 and $8.5 \mathrm{~nm}$. In general, the shape of the curves is similar to other commercial CPCs as presented in Hermann et al. (2007), Petäjä et al. (2006) and Wiedensohler et al. (1997).

As shown in Wang et al. (2002), the ratio between aerosol and saturator flow also influences the measurement performance of the FCPC. To investigate this in detail, one particle diameter from the cutoff region was selected for each temperature difference and the aerosol flow was varied between 0.1 and $0.41 \mathrm{~min}^{-1}$. The flow of $11 \mathrm{~min}^{-1}$ through the optics was kept constant during all measurements. The

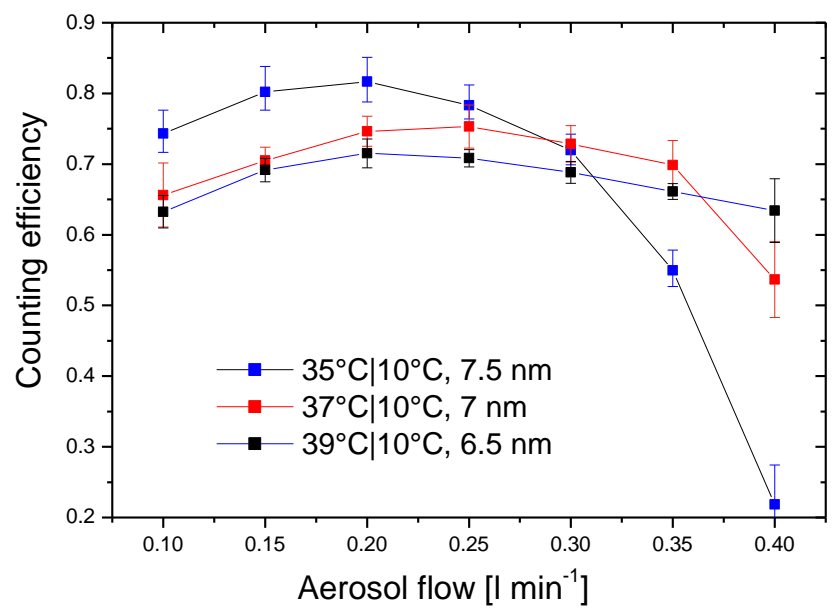

Fig. 3. Counting efficiency of the Fast CPC at different temperature differences and different aerosol flows compared to the electrometer (TSI 3068B) for selected particle diameters. The total flow (saturator + aerosol flow) was kept constant at $11 \mathrm{~min}^{-1}$. Squares present the mean measurement points, the error bars the standard deviation.

Table 1. Fit parameters $a, D_{1}$ and $D_{2}$ for Eq. (1) as well as the $50 \%$ detection diameter $D_{\text {P50 }}$ of the mean FCPC counting efficiency curves as presented in Fig. 2.

\begin{tabular}{lccccc}
\hline$T_{\mathrm{S}}\left[{ }^{\circ} \mathrm{C}\right]$ & $T_{\mathrm{C}}\left[{ }^{\circ} \mathrm{C}\right]$ & $a$ & $D_{1}$ & $D_{2}$ & $D_{\mathrm{P} 50}[\mathrm{~nm}]$ \\
\hline 39 & 10 & 1.00 & 5.5 & 1.1 & 6.1 \\
37 & 10 & 0.99 & 5.6 & 1.9 & 7.1 \\
35 & 10 & 0.98 & 6.7 & 2.5 & 8.5 \\
\hline
\end{tabular}

results are presented in Fig. 3. The squares present the mean measurement points, the error bars the standard deviation. Each temperature difference has a maximum counting efficiency region between 0.15 and $0.301 \mathrm{~min}^{-1}$, for $37^{\circ} \mathrm{C} \mid 10^{\circ} \mathrm{C}$ and $39^{\circ} \mathrm{C} \mid 10^{\circ} \mathrm{C}$ the variation between these aerosol flow settings causes less than $10 \%$ variation in the counting efficiency. This is a good result, because the FCPC seems to be relatively insensitive compared to small fluctuations in the flow ratio. The definition of the optimum aerosol flow also influences the counting statistics. Thus, the lower the aerosol flow is, the fewer particles are counted, resulting in higher uncertainties and lower time resolution. Thus, for the presented instrument a compromise between counting efficiency and statistics was selected to define the aerosol flow to $0.31 \mathrm{~min}^{-1}$, which is still in the maximum region for $37^{\circ} \mathrm{C} \mid 10^{\circ} \mathrm{C}$ and $39^{\circ} \mathrm{C} \mid 10^{\circ} \mathrm{C}$ being the preferred temperature differences. For an operation at $35^{\circ} \mathrm{C} \mid 10^{\circ} \mathrm{C}$ the aerosol flow might be decreased to $0.251 \mathrm{~min}^{-1}$. 


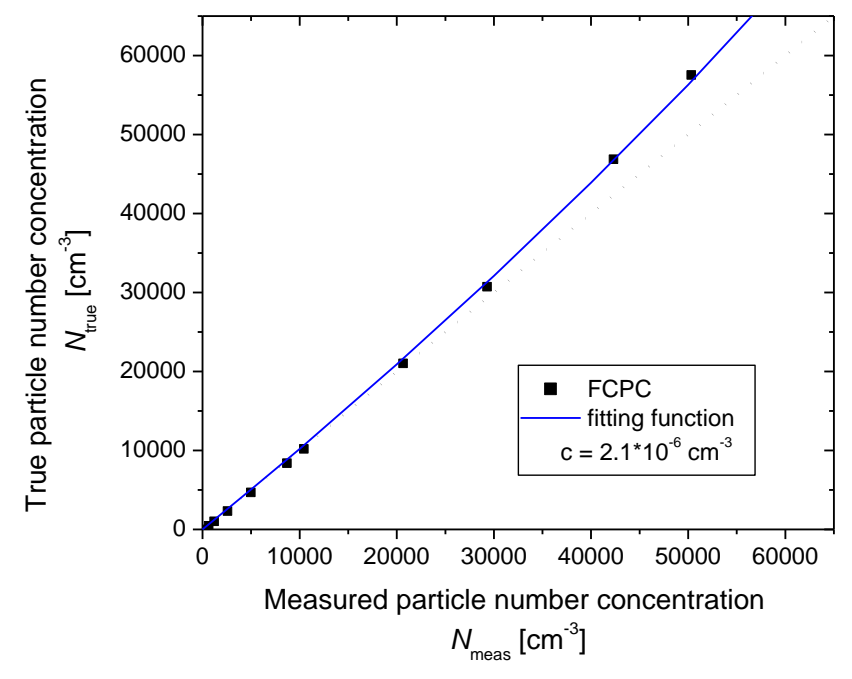

Fig. 4. Measurements of the FCPC and EM at different concentrations (squares) as well as a fitted curve according to Eq. (2).

\subsection{Particle coincidence}

The calibration setup was used to also investigate the effect of a coincidence on the measured number concentration measured by the new FCPC. For this purpose $30-n m$ particles were generated with different number concentrations between 100 and $58000 \mathrm{~cm}^{-3}$. FCPC and EM measured in parallel at each selected concentration, while the EM is considered to give the true particle number concentration $N_{\text {true }}$. The relation between true and measured number concentration influenced by coincidence is given in the CPC-manuals (e.g., TSI Operation and service manual for CPC 3771/3772) as:

$N_{\text {true }}=N_{\text {meas }} \exp \left(N_{\text {true }} \cdot c\right)$.

Here, $c$ is the coincidence parameter which is given by the product of the volume flow rate and the residence time within the viewing volume of the CPC optics. The measurement results from EM and FCPC are displayed in Fig. 4. To correct measured data for coincidence, a function is fitted to the measurement points according to Hermann and Wiedensohler (2001), resulting in a $c$ of $2.1 \times 10^{-6} \mathrm{~cm}^{-3}$. To calculate $c$ from theory, we use the volume flow rate of $0.31 \mathrm{~min}^{-1}$ and the residence time in the viewing volume of $0.35 \mu$ s (cf. TSI Operation and service manual for CPC 3771/3772) resulting in a theoretical value for $c$ of $1.75 \times 10^{-6} \mathrm{~cm}^{-3}$ which is close to the value obtained from measurements above. This demonstrates that the coincidence of the FCPC, because of its low volume flow rate, is lower than for the unsheathed commercial CPCs such as TSI 3772 and the operation range can be easily extended to concentrations up to $60000 \mathrm{~cm}^{-3}$.
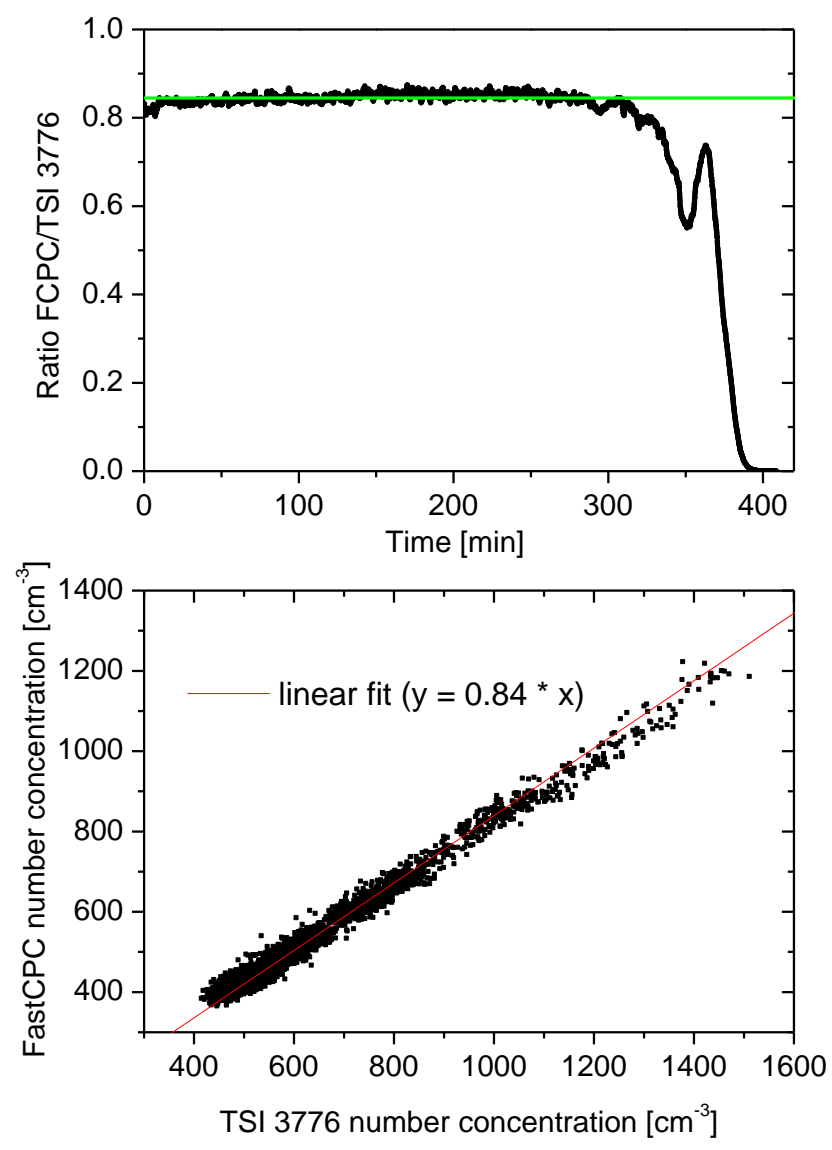

Fig. 5. Comparison of FCPC and TSI 3776 sampling room air from the laboratory. The upper plot displays the time series of the ratio number concentrations FCPC/TSI 3776. The horizontal green line displays the average value (0.85) during the first $250 \mathrm{~min}$. The lower plot shows the direct comparison of the number concentration measurement for the first $250 \mathrm{~min}$ as well as a linear fit.

\subsection{Counting stability}

In addition to the size-dependent counting efficiency, the measurement stability of a particle counter had to be investigated. Because of the deployed mixing, this is not a trivial question. The presented FCPC is intended to measure onboard the helicopter-borne platform ACTOS (Siebert et al., 2006), where measurement flights typically last two hours. Thus, stable particle counting over this period, at least, is required for any deployed instrumentation. To test the stability, various measurements were performed in the laboratory.

First, the FCPC was operated over a few hours in parallel with a TSI CPC 3776 under laboratory conditions. Figure 5, upper graph, shows the evolution of the ratio FCPC/TSICPC 3776 within $7 \mathrm{~h}$ measuring ambient air from the laboratory. The FCPC was operated with $T_{\mathrm{S}}=37^{\circ} \mathrm{C}$ and $T_{\mathrm{C}}=10^{\circ} \mathrm{C}$ and butanol was added into the saturator only prior to the measurement. In fact, the ratio between both CPCs was stable for $5 \mathrm{~h}$, which is sufficient for the desired 


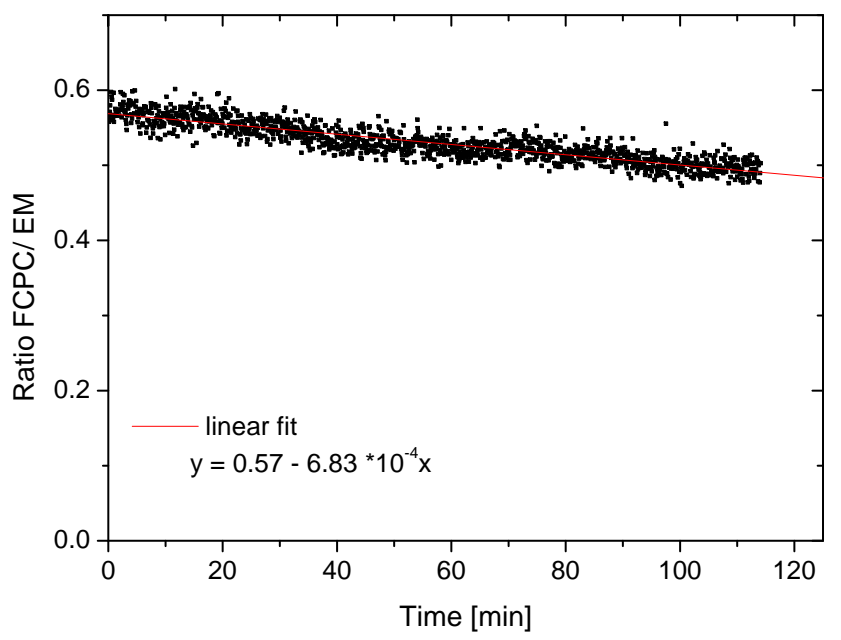

Fig. 6. Counting efficiency of the FCPC in comparison with the electrometer (ratio FCPC/EM) measuring 8-nm silver particles at $37^{\circ} \mathrm{C} \mid 10^{\circ} \mathrm{C}$. The red line displays the linear trend.

flight endurance, with an average concentration ratio of 0.85 . Both CPCs have a different $D_{\text {P50 }}$ which would lead to different total number concentrations if the fraction of particles between 3 and $7 \mathrm{~nm}$ varies. There was no source for ultrafine particles active in the laboratory during the test, and the total concentration decreased from $1300 \mathrm{~cm}^{-3}$ to $500 \mathrm{~cm}^{-3}$ during the first $150 \mathrm{~min}$. There was no measurement of number size distributions available to quantify the change during the observed period. However, we expect that the number concentration between 3 and $7 \mathrm{~nm}$ decreased, because there was no source for such small particles and their lifetime is short. This can also be seen from a slightly positive trend in the first part of the observed period (Fig. 5, upper graph). The lower graph of Fig. 5 shows the scatter plot of measured number concentrations during the first $250 \mathrm{~min}$. Obviously measurements from both counters are well correlated $\left(R^{2}=0.98\right)$, the standard deviation from the linear trend is $\sim 19$ (square root of the mean error). After $6 \mathrm{~h}$ of continuous operation, the counting efficiency dropped significantly caused by the smaller butanol reservoir of the FCPC compared to the TSI CPC 3776.

In addition to the comparison of total number concentrations, the stability at individual particle sizes was investigated. With regard to the counting efficiency curve (Fig. 2), measurements at individual diameters were performed: (1) diameters on the slope of the counting efficiency curve (close to $D_{\mathrm{P} 50}$ ), to check how stable the location of $D_{\mathrm{P} 50}$ is, and (2) diameters above the cutoff region, i.e., where the maximum counting efficiency was reached. For laboratory-generated, monodisperse particles the EM is the most reliable reference instrument and was, therefore, used for further measurements.

Figure 6 shows the ratio of number concentrations measured by the FCPC and the EM for 8-nm silver particles over
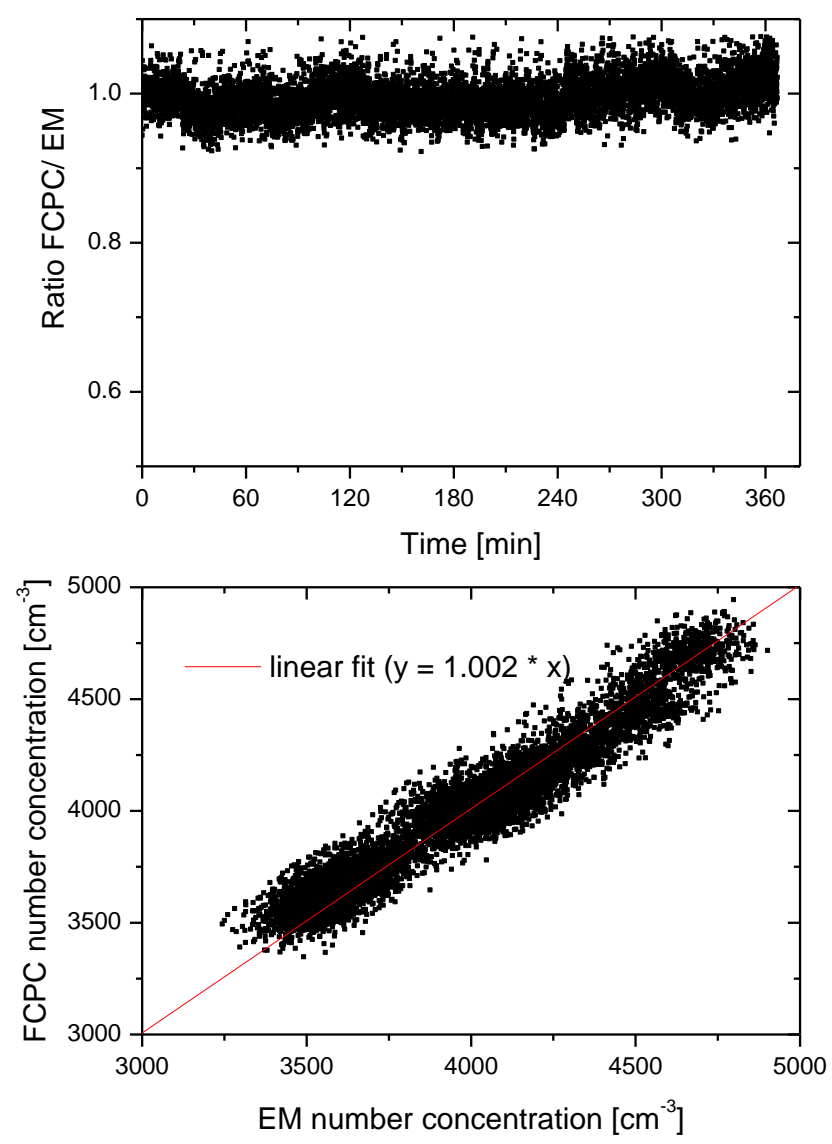

Fig. 7. Counting efficiency of the FCPC in comparison with the electrometer (ratio FCPC/EM) measuring 30-nm silver particles at $37^{\circ} \mathrm{C} \mid 10^{\circ} \mathrm{C}$ : the upper plot shows the ratio FCPC/EM, the lower one the corresponding scatter plot of the number concentrations. The red line displays the linear trend in the lower plot.

$2 \mathrm{~h}$. The FCPC was operated at $T_{\mathrm{S}}\left|T_{\mathrm{C}}=37^{\circ} \mathrm{C}\right| 10^{\circ} \mathrm{C}$. Consequently $8 \mathrm{~nm}$ correspond to the steep part of the counting efficiency curve ( $\eta \approx 0.6$, cf. Fig. 2$)$. The FCPC/EM ratio decreased during that time by approximately 0.08 or $13 \%$ from the initial value, resulting from the linear trend which is fitted to the data. Further measurements with diameters in the cutoff region of the counting efficiency curve gave a similar picture. This decrease in counting efficiency is probably due to the decreasing butanol reserve within the saturator of the FCPC. Thus, either the decreasing butanol reserve decreases the counting efficiency in general or it shifts only the 50\%detection efficiency diameter towards larger diameters. To prove this, measurements at $30 \mathrm{~nm}$ were performed, where the counting efficiency is at its maximum value.

Figure 7 shows the ratio of number concentrations measured by the FCPC and the EM for 30-nm silver particles (upper plot), and the corresponding scatter plot of the absolute concentration values (lower plot). The FCPC was operated again at $T_{\mathrm{S}}\left|T_{\mathrm{C}}=37^{\circ} \mathrm{C}\right| 10^{\circ} \mathrm{C}$. The mean ratio is stable over six hours and a trend is not recognizable. The lower 
plot illustrates that both measurements are well correlated $\left(R^{2}=0.94\right)$, the standard deviation from the linear trend is $81 \mathrm{~cm}^{-3}$.

In summary, it was shown that the counting efficiency at $30 \mathrm{~nm}$ was stable while the counting efficiency at $8 \mathrm{~nm}$ decreased by $13 \%$ over $2 \mathrm{~h}$ of continuous operation. From these results, we can conclude that a decrease in the butanol content causes a shift in the counting efficiency curve to larger diameters. The shape of the curve as well as the maximum counting efficiency is stable. Thus, a decreasing butanol reservoir has an effect on the counting efficiency mainly in the cutoff region $(\sim 6-12 \mathrm{~nm})$ including the $D_{\mathrm{P} 50}$. The $13 \%$ decrease at $8 \mathrm{~nm}$ within $2 \mathrm{~h}$ results in a shift of approximately $0.5 \mathrm{~nm}$ to larger diameters. A diameter shift of $0.5 \mathrm{~nm}$ is probably similar to the uncertainty of the DMA size selection due to deviations from the ideal flow rates as well as those due to skewing of the initial size distribution in connection with the width of the transfer function.

\subsection{Time response: experiments under atmospheric conditions}

The time response of CPCs was investigated in previous studies by different techniques. Mostly fast-reacting valves in laboratory setups were applied (e.g., Held and Klemm, 2006) or the statistical behaviour of natural fluctuations of particle concentrations due to atmospheric turbulence was analysed (Buzorius, 2001).

Our approach is taking advantage of the combination of atmospheric turbulence near ground and a strong isolated particle source, which results in strong fluctuations due to sharp gradients in the particle number concentration. Such strong gradients are usually not observed in the atmosphere, but the resulting conditions provide a perfect environment estimating the time response of the new FCPC. The following analysis is two-fold: (i) the structure of a sharp ramp is analysed to estimate the typical time response, and (ii) power spectral analysis is used to distinguish between coherent structures of particle concentration in a turbulent flow field and white noise of purely Poisson distributed particles. Coherent structures will result in a power spectrum with a slope of approximately " $-5 / 3$ " (so-called "inertial subrange behavior", Wyngaard, 2010), whereas white noise will result in a flat spectrum. The latter can be due to increasing sampling noise which means that not enough particles are counted within the sampling gate or that the time response of the FCPC is too low to resolve the coherent structures.

\subsubsection{Time series analysis}

Figure 8 shows a 800-s long record of the particle number concentration $N$ measured with the FCPC under atmospheric conditions. The FCPC was placed at a height of $\sim 1 \mathrm{~m}$ above ground, a gasoline power generator was running as a particle point source $5 \mathrm{~m}$ upstream of the measurement location. The mean wind speed was about $2-3 \mathrm{~m} \mathrm{~s}^{-1}$ with slightly varying direction. The sampling gate of the data acquisition system was set to $10 \mathrm{~ms}$ which implies a sampling frequency of $100 \mathrm{~Hz}$.

The time series indicates strong fluctuations of $N$ with the amplitude of $N$ ranges from about 1 to $3 \times 10^{4} \mathrm{~cm}^{-3}$ for the atmospheric background superimposed by concentrations of up to $8 \times 10^{4} \mathrm{~cm}^{-3}$ representative for the exhaust of the power generator.

A 25-s long subrecord is shown in the lower panel and highlights the sharp ramp structures in the time series at the edges of the "exhaust events". Such sharp ramps will now be used to estimate the typical time response of the FCPC.

It is widely common to describe the temporal behaviour of a CPC as a "first-order response system", which means the change of the measured concentration $d N_{i} / d t$ is proportional to the difference $\left(\delta N=N_{0}-N_{i}\right.$ ) between the true concentration $N_{0}$ and the measured concentration $N_{i}$. Assuming the true concentration exhibits a step function at time $t_{0}$ and jumps from $N_{0}$ to $N_{1}$ the measured concentration can be described by:

$N_{i}(t)=N_{1}+\left(N_{0}-N_{1}\right) \cdot \exp \left(\frac{-\left(t-t_{0}\right)}{\tau}\right)$,

for $t>t_{0}$ and with $\tau$ is the mixing time of the FCPC.

Figure 9 shows a 120 -ms long subrecord including a sharp ramp which is marked by the blue box in Fig. 8. The change of the particle number concentration $\delta N$ is about $6 \times 10^{4} \mathrm{~cm}^{-3}$. It has to be pointed out that the gate width of $10 \mathrm{~ms}$ which was chosen for technical reasons is not sufficient to fully resolve this jump. Therefore, it is not possible to define $t_{0}$ exactly. Using $\tau=5 \mathrm{~ms}$ and $t_{0}=660.275 \mathrm{~s}$, Eq. (3) yields a function which best fits with the observations (see dashed green line in Fig. 9). However, with this observation and in particular the long gate of $10 \mathrm{~ms}$, the mixing time $\tau$ can only be roughly estimated. For comparison, the mixing time for TSI CPC 3010, being a comparable also unsheathed instrument, was measured in different studies and is between 0.77 and $0.96 \mathrm{~s}$ (Buzorius, 2001; Held and Klemm, 2006), which is approximately a factor of 50 to 200 higher compared to the new FCPC.

\subsubsection{Spectral behaviour}

The estimated mixing time is a parameter which only describes the technical response of the FCPC. However, the question of how fast the FCPC can measure under atmospheric conditions depends also on the counting process itself. If the number of counted particles is low, the statistical uncertainty of the counting process might mask coherent structures which are present in the atmosphere.

In order to distinguish between the coherent structures in the turbulent concentration field and white noise of purely Poisson distributed particles, spectral analysis was applied. From a measured time series $N(t)$ power spectral density 


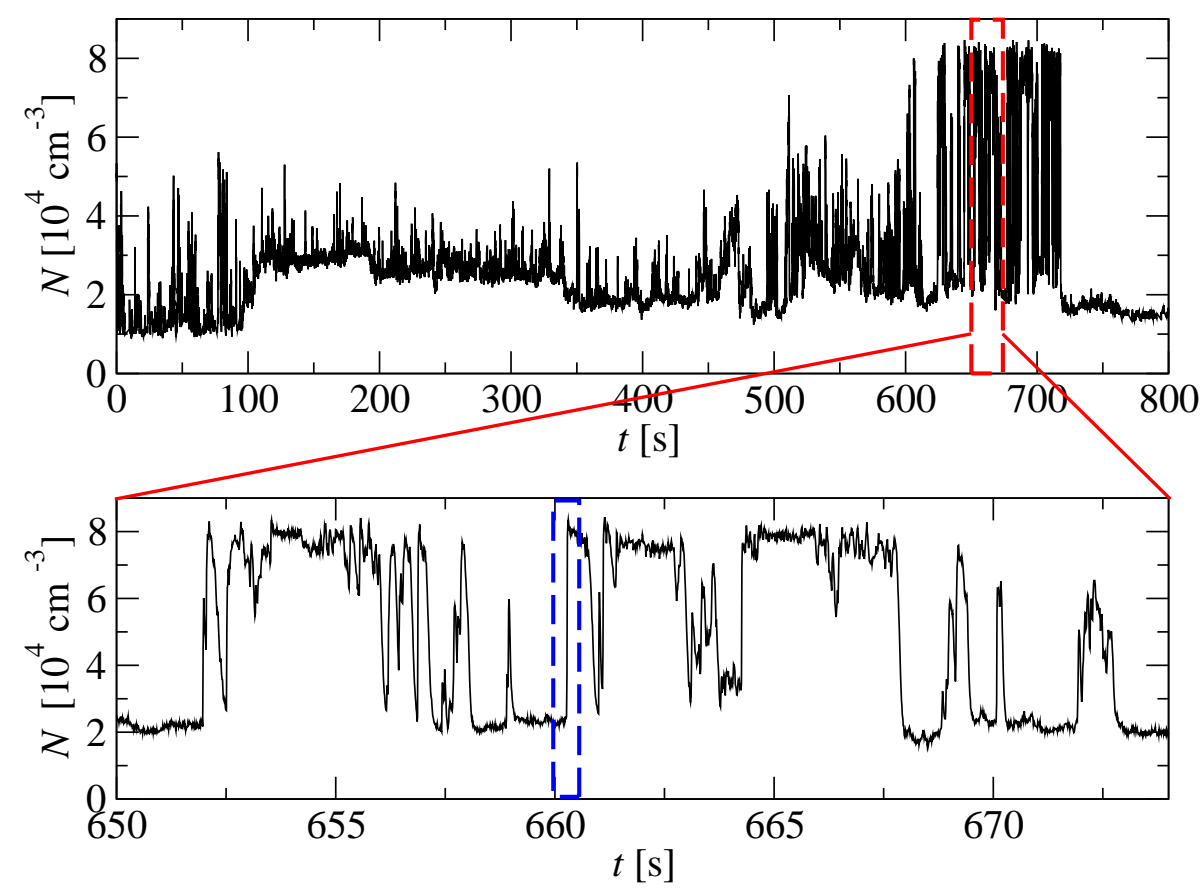

Fig. 8. Time series of particle number concentration $N$ measured with the FCPC under atmospheric conditions, about $5 \mathrm{~m}$ downstream of an artificial particle source (gasoline power generator). The sampling gate was $10 \mathrm{~ms}$. At the end of the record $(t \sim 600-720 \mathrm{~s})$, the power generator was running out of gasoline resulting in an increased production of particles. The lower panel shows an enlarged portion of the record with strong variation of $N$ due to the fluctuations of the wind direction and the particle production of the power generator. The blue box depicts a sharp ramp analysed in Fig. 9.

functions (PSD) were estimated by using a Fast Fourier Transformation (FFT). That is, the variance of $N$ per frequency interval is displayed as a function of frequency $f$. From turbulence theory (e.g., Wyngaard, 2010, and references therein) it is known that the PSD of a turbulent time series results in a spectrum which is proportional to approximately $f^{-5 / 3}$ in the so-called inertial subrange. For atmospheric conditions, this inertial subrange is from about a few millimetres up to the largest eddies in the atmosphere. For ground-based measurements in a height $h$ the largest eddies which contribute to the measured time series have a length scale of $l \approx 4 \cdot h$ (see Fig. 1 in Muschinski and Roth, 1994, 1994 for an explanation) which defines the upper limit for the inertial subrange. With the relation $f=U / l$, where $U$ is the mean flow velocity one can estimate the low frequency end $f_{0}$ for the inertial subrange: $f_{0}=U /(4 \cdot h)$.

It should be noted that classical inertial subrange scaling - PSD $\propto f^{-5 / 3}$ - is based on assumptions such as a quasihomogeneous and local isotropic velocity field and there is no thorough discussion in the literature why a time series based on a counting process should also follow exactly the same scaling laws as, for instance, the wind velocity or temperature. A detailed discussion about this issue and possible deviations from the classical inertial subrange scaling is going beyond the scope of this work. We use the spectral analysis only to distinguish between scaling regions (e.g., frequency ranges with PSD $\propto f^{-\alpha}$ where $\alpha$ is the scaling exponent) and white noise which results in a flat spectrum meaning that all the variance of the fluctuations is equally distributed over the frequency range.

The following analysis is based on a 5-min long dataset taken about $1 \mathrm{~m}$ above ground level. The mean wind speed was about $U=3-4 \mathrm{~m} \mathrm{~s}^{-1}$ which resulted in $f_{0} \approx 3 \mathrm{~m} \mathrm{~s}^{-1} /(4 \cdot 1 \mathrm{~m})=0.75 \mathrm{~Hz}$. That is, for a frequency range between $0.75 \mathrm{~Hz}$ up to the maximum frequency of our measurements $(100 \mathrm{~Hz})$ we can safely conclude that our particle measurements are well within the inertial subrange.

The ground surface was flat for at least $200 \mathrm{~m}$ up-stream to the measurement point without any artificial sources for aerosol particles. The sampling gate was again $10 \mathrm{~ms}$. Figure 10 shows the PSD of $N$ in a log-log representation; the black dashed line indicates the $-5 / 3$ slope as a reference for inertial subrange scaling, the red dot-dashed line is a PSD based on artificial data which are completely Poisson distributed resulting in a flat spectrum. The increased scatter at the low-frequency end is due to statistical noise since only a few events with a time scale of $t=1 / f \approx 1 / 0.01 \mathrm{~Hz}=100 \mathrm{~s}$ can be resolved in 300 s-long time series.

The black curve with circles represents the PSD with full resolution of $f_{\mathrm{s}}=100 \mathrm{~Hz}$ (note that the PSD can be displayed only up to the so-called Nyquist frequency $f_{\mathrm{Ny}}=f_{\mathrm{s}} / 2=50 \mathrm{~Hz}$ ). In the frequency range between $10^{-2}$ 


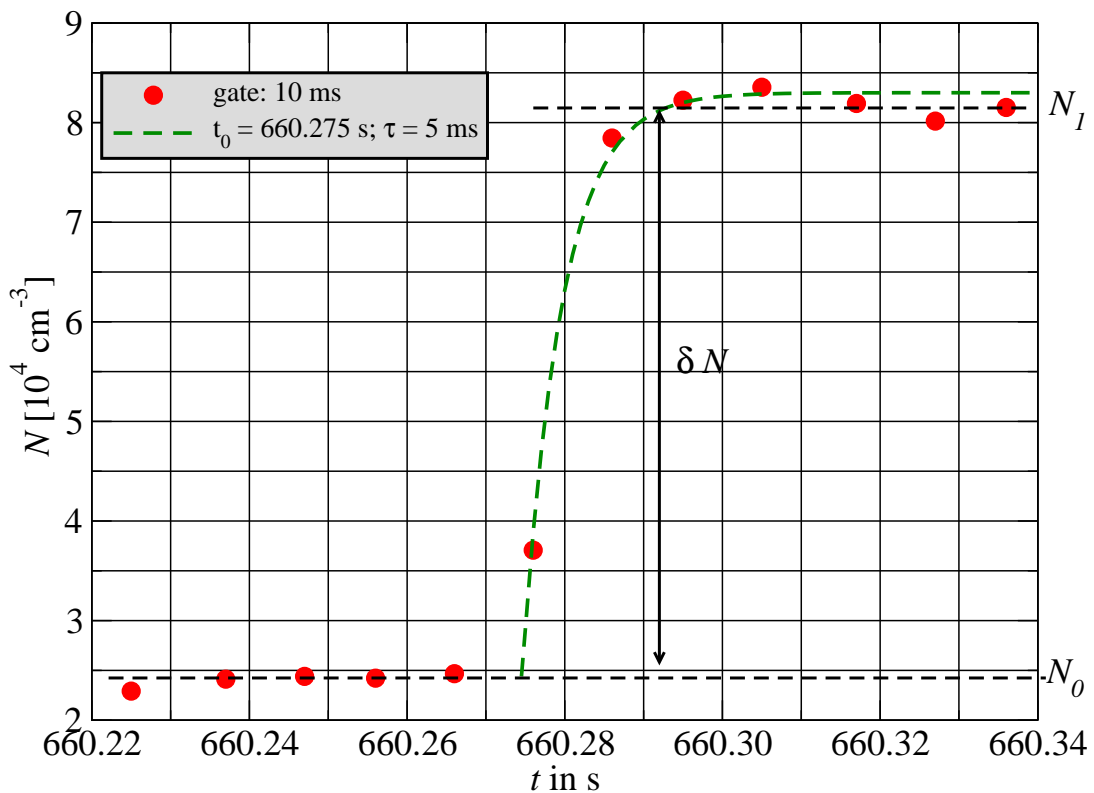

Fig. 9. A 120-ms long subrecord of the time series presented in Fig. 8 marked by the blue box is shown. The varying wind direction created strong fluctuations of $N$ resulting in sharp ramps. Assuming a first-order response of the FCPC, the e-folding time $\tau$ can be estimated from such a ramp to $\tau \approx 5 \mathrm{~ms}$.
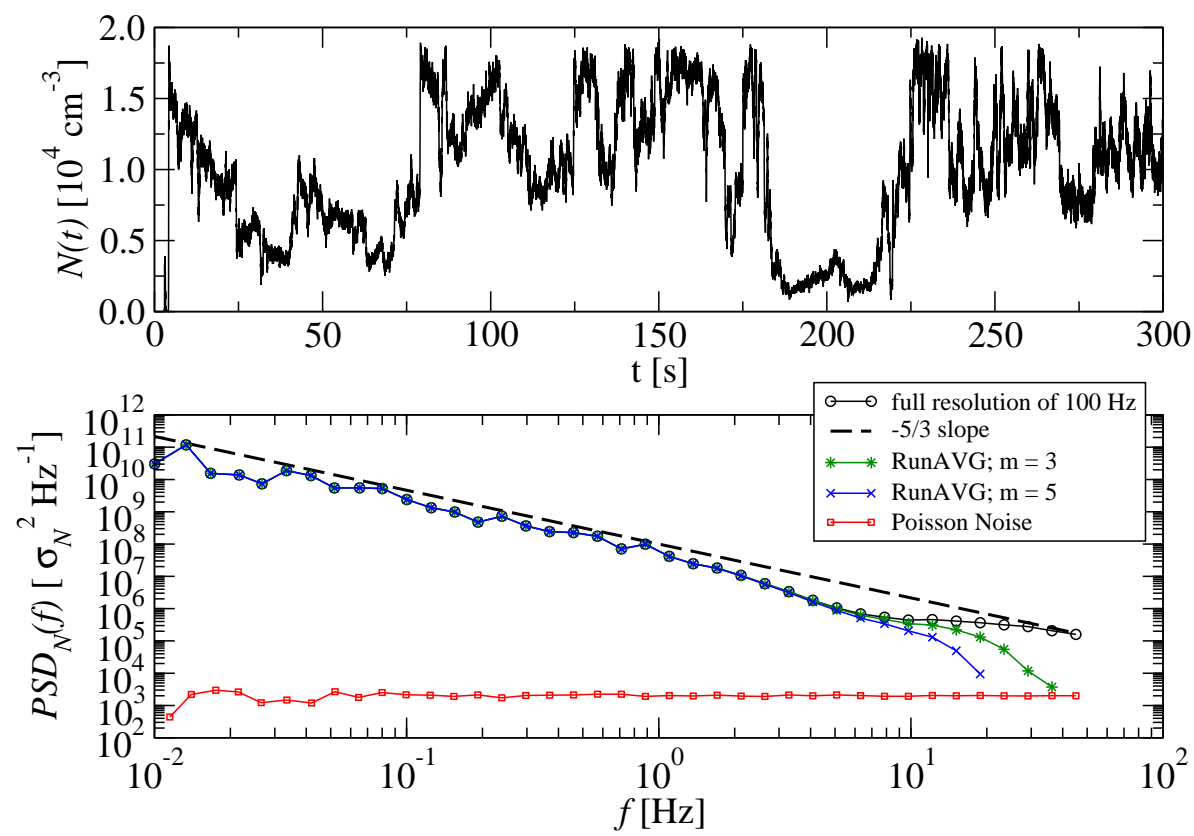

Fig. 10. The upper panel shows a 300-s long time series recorded under undisturbed atmospheric conditions. The FCPC was located about $1 \mathrm{~m}$ a.g.l. (above ground level). The lower panel shows corresponding power spectral density functions using the full resolution (black curve), a running mean over 3 values (green curve) and a running mean over 5 values (blue curve). The red curve shows the spectral density of Poisson distributed data resulting in white noise (flat line). The black dashed line indicates a $-5 / 3$ slope for inertial subrange scaling.

and $1 \mathrm{~Hz}$, the PSD shows roughly a $-5 / 3$ slope with increased scatter in particular for the lowest frequencies due to statistical noise. For higher frequencies the PSD shows less scatter, but a steeper slope compared with $-5 / 3$. At a frequency of $\approx 6 \mathrm{~Hz}$ the PSD begins to flatten which indicates that the Poisson noise dominates over the coherent structures.
In order to reduce the sampling noise, a running average with a window width $m$ was applied to the time series. Here $m=3$ (green curve) and $m=5$ (blue curve) were chosen. That is, the sampling gate was increased from $10 \mathrm{~ms}$ to 30 and $50 \mathrm{~ms}$, respectively. This kind of "low-pass filtering" with a cut-off frequency of $f_{\mathrm{c}} \approx f_{\mathrm{s}} /(2 \mathrm{~m})$ reduces the sampling 
noise and, therefore, increases the scaling range: most obvious for $m=5\left(f_{\mathrm{c}}=10 \mathrm{~Hz}\right)$, the scaling range can be expanded from about 6 to $12 \mathrm{~Hz}$. Consequently, under these atmospheric conditions the FCPC has a realistic time resolution of approximately $0.1 \mathrm{~s}$.

\section{Summary and outlook}

A new fast-measuring CPC was built based on the setup of Wang et al. (2002). The time resolution of this instrument is significantly higher than that of commercially available instruments, therefore, it is called Fast CPC (FCPC). The functionality and stable operation of the FCPC was demonstrated in the laboratory as well as under atmospheric conditions for the first time.

The counting efficiency shows a behaviour similar to laminar flow-type CPCs: with increasing temperature difference $D_{\mathrm{P} 50}$ is moving to smaller diameters. In this study, temperature differences from 25 to $29 \mathrm{~K}$ were chosen leading to a $D_{\text {P50 }}$ between 8.5 and $6.1 \mathrm{~nm}$. Above $25 \mathrm{~nm}$ the counting efficiency reached its maximum value of $98-100 \%$ compared to an aerosol electrometer, a value which is similar to other commercial CPCs. The FCPC demonstrated its ability to perform stable measurements over a few hours, except that the $D_{\text {P50 }}$ of the counting efficiency curve is moving to larger particle sizes when butanol is not filled continuously. A decrease of $13 \%$ at a fixed size on the slope corresponds to a shift of less than $0.5 \mathrm{~nm}$, which defines the error in diameter of our efficiency curve. Considering the total number in the concentration only, without a dominant nucleation mode, the shift is negligible. For detailed studies on new particle formation, as well as potential ground-based or long-term applications, the FCPC needs to be modified to ensure a continuous butanol supply into the wick. As proposed, the current version fulfils the requirements of a two-hour measurement flight of the helicopter-borne platform ACTOS.

The usable time resolution of the FCPC for atmospheric measurements is limited by two facts: (i) the mixing time of the FCPC which was estimated to be on the order of $5 \mathrm{~ms}$ and which is fully determined by technical issues, and (ii) the crossover of coherent turbulent structures and Poisson noise which can be derived from spectral analysis. The latter one is completely defined by the width of the sampling gate, the particle number concentration and fluctuations and the atmospheric turbulence and was estimated to approximately $0.1 \mathrm{~s}$ in the presented case.

The new FCPC was implemented on the helicopter-borne platform ACTOS and applied in boundary layer measurements. The focus here is on the analysis of particle number fluctuations in regions with highly turbulent structures such as cloud edges and mixing zones near inversions. The FCPC enables airborne particle measurements on the meter-scale for the first time which will improve the understanding of small-scale particle processes significantly.
Acknowledgements. The authors thank Michael Eckinger and Christian Kraft for their assistance in the laboratory, i.e., the calibration and modification of different versions of the FCPC. The project was supported by the German Science Foundation DFG (WE 2757/1-1).

Edited by: M. Wendisch

\section{References}

Banse, D. F., Esfeld, K., Hermann, M., Sierau, B., and Wiedensohler, A.: Particle counting efficiency of the TSI CPC 3762 for different operating parameters, J. Aerosol Sci., 32, 157-161, 2001.

Baron, P. A. and Willeke, K.: Aerosol Measurements: Principles Techniques and Applications, John Wiley and Sons, New York, 1131 pp., 2001.

Buzorius, G.: Cut-off sizes and time constants of the CPC TSI 3010 operating at 1-3 lpm flow rate, Aerosol Sci. Tech., 35, 577-585, 2001.

Buzorius, G., Rannik, Ü., Mäkelä, J. M., Vesala, T., and Kulmala, M.: Vertical aerosol particle fluxes measured by eddy covariance technique using condensational particle counters, J. Aerosol Sci., 129, 157-171, 1998.

Buzorius, G., Rannik, Ü., Nilsson, D., and Kulmala, M.: Vertical fluxes and micrometeorology during aerosol particle formation events, Tellus B, 53, 394-405, 2001.

Held, A. and Klemm, O.: Direct measurement of turbulent particle exchange with a twin CPC eddy covariance system, Atmos. Environ., 40, S92-S102, 2006.

Hermann, M. and Wiedensohler, A.: Counting efficiency of condensation particle counters at low-pressures with illustrative data from the upper troposphere, J. Aerosol Sci., 32, 975-991, 2001.

Hermann, M., Wehner, B., Bischof, O., Han, H.-S., Krinke, T., Liu, W., Zerrath, A., and Wiedensohler, A.: Particle counting efficiencies of new TSI condensation particle counters, J. Aerosol Sci., 38, 674-682, 2007.

Hoppel, W. A., Frick, G. M., Fitzgerald, J. W., and Laerson, R. E.: Marine boundary layer measurements of new particle formation and the effects nonprecipitating clouds have on aerosol size distribution, J. Geophys. Res., 99(D7), 14443-14459, 1994.

Klemm, O., Held, A., Forkel, R., Gasche, R., Kanter, H. J., Rappenglueck, B., Steinbrecher, R., Müller, K., Plewka, A., Cojocariu, C., Kreuzwieser, J., Valverde-Canossa, J., Schuster, G., Moortgat, G. K., Graus, M., and Hansel, A.: Experiments on forest/amtosphere exchange: Climatology and fluxes during two summer campaigns in NE Bavaria, Atmos. Environ., 40, S3-S20, 2006.

Muschinski, A. and R. Roth: What does a Large-Eddy simulation simulate?, Contr. Atmos. Phys., 66, 335-346, 1994.

Okuyama, K., Kousaka, Y., and Moutouchi, T.: Condensational growth of ultrafine aerosol-particles in a new particle-size magnifier, Aerosol Sci. Tech., 3, 353-366, 1984.

Petäjä, T., Mordas, G., Manninen, H., Aalto, P. P., Hämeri, K., Kulmala, M.: Detection efficiency of a water-based TSI condensation particle counter 3785, Aerosol Sci. Tech., 40, 1090-1097, 2006. 
Quant, F. R., Caldow, R., Sem, G. J., and Addison, T. J.: Performance of condensation particles counters with three continuousflow designs, Aerosol Sci. Tech., 23, S405-S408, 1992

Scheibel, H. G. and Porstendörfer, J.: Generation of monodisperse $\mathrm{Ag}-$ and $\mathrm{NaCl}$ aerosols with particle diameters between 2 and 300 nm, J. Aerosol Sci., 14, 113-126, 1983.

Siebert, H., Stratmann, F., and Wehner, B.: First observations of increased ultrafine particle number concentrations near the inversion of a continental planetary boundary layer and its relation to ground-based measurements, Geophys. Res. Lett., 31, L09102, doi:10.1029/2003GL019086, 2004.

Siebert, H., Franke, H., Lehmann, K., Maser, R., Saw, E. W., Schell, D., Shaw, R. A., and Wendisch, M.: Probing fine-scale dynamics and microphysics of clouds with helicopter-borne measurements, B. Am. Meteorol. Soc., 87, 1727-1738, 2006.

Stratmann, F., Siebert, H., Spindler, G., Wehner, B., Althausen, D., Heintzenberg, J., Hellmuth, O., Rinke, R., Schmieder, U., Seidel, C., Tuch, T., Uhrner, U., Wiedensohler, A., Wandinger, U., Wendisch, M., Schell, D., and Stohl, A.: New-particle formation events in a continental boundary layer: first results from the SATURN experiment, Atmos. Chem. Phys., 3, 1445-1459, doi:10.5194/acp-3-1445-2003, 2003.

Vanhanen, J., Mikkilä, J., Lehtipalo, K., Sipilä, M., Manninen, H. E., Siivola, E., Petäjä, T., and Kulmala, M.: Particle Size Magnifier for Nano-CN Detection, Aerosol Sci. Tech., 45, 533-542, 2011.
Wang, J., McNeill, V. F., Collins, D. R., and Flagan, R. C.: Fast mixing condensation nucleus counter: Application to rapid scanning differential mobility analyzer measurements, Aerosol Sci. Tech., 36, 678-689, 2002.

Weber, R. J., Chen, G., Davis, D. D., Mauldin III, R. L., Tanner, D. J., Eisele, F. L., Clarke, A. D., Thornton, D. C., and Bandy, A. R.: Measurements of enhanced $\mathrm{H}_{2} \mathrm{SO}_{4}$ and 3-4 nm particles near a frontal cloud during First Aerosol Characterisation Experiment (ACE 1), J. Geophys. Res., 106(D20), 24107-24117, 2001.

Wehner, B., Siebert, H., Ansmann, A., Ditas, F., Seifert, P., Stratmann, F., Wiedensohler, A., Apituley, A., Shaw, R. A., Manninen, H. E., and Kulmala, M.: Observations of turbulence-induced new particle formation in the residual layer, Atmos. Chem. Phys., 10, 4319-4330, doi:10.5194/acp-10-4319-2010, 2010.

Wiedensohler, A.: An approximation of the bipolar charge distribution for particles in the submicron size range, J. Aerosol Sci., 19, 387-389, 1988.

Wiedensohler, A., Orsini, D., Covert, D. S., Coffman, D., Cantrell, W., Havlicek, M., Brechtel, F. J., Russell, L. M., Weber, R. J., Gras, J., Hudson, J. G., and Litchy, M.: Intercomparison study of the size-dependent counting efficiency of 26 condensation particle counters, Aerosol Sci. Tech., 27(2), 224-242, 1997.

Wyngaard, J. C.: Turbulence in the atmosphere, Cambridge University Press, 408p., 2010. 